\title{
Diagnostic des ouvrages portuaires du département des Pyrénées-Atlantiques
}

\author{
Jean-Marc Beynet ${ }^{1}$, Jean Bougis ${ }^{2}$, A. Jouandet ${ }^{3}$ et A. Roudil ${ }^{4}$ \\ ${ }^{1}$ Directeur de projet, BRL Ingénierie, 1105, Avenue P.Mendès-France, \\ 30001 Nîmes Cedex, Tél. : 04.66.87.51.14, jean-marc.beynet@brl.fr \\ ${ }^{2}$ Ingénieur Conseil, 34 Chemin du Moulin, 06650 OPIO, \\ Tél.:04.93.77.74.22,jbougis@aws.fr \\ ${ }^{3}$ Technicien Territorial, Conseil Général des Pyrénées Atlantiques, \\ DAEE,64100Bayonne,Tél :05.59.46.51.48, jouandet@.cg64.fr \\ ${ }^{4}$ Contrôleur Principal des TPE, DDE, Subdivision de Saint Jean de \\ Luz, 64500 Ciboure, Tél : 05.59.47.10.45
}

Résumé

Cet article présente les études de diagnostic ainsi que la mise en cuvre du programme pluriannuel de remise en état que le Conseil Général des PyrénéesAtlantiques a engagé à partir de 1998, sur l'ensemble de ses ouvrages portuaires. Après un rappel sur les conditions d'exposition à la houle des ports concernés, les principaux problèmes observés sur les ouvrages sont présentés avec les différents critères qui ont été retenus pour programmer la remise en état. Le point est ensuite fait sur l'état des travaux entrepris.

Abstract

This paper describes the diagnostic studies and the implementation of the harbour structures rehabilitation, carried out from 1998 by the Departmental Council of the Pyrénées-Atlantiques. Following a remind on swell conditions of the harbours understudy, main problems on structures themselves are listed, together with the criteria which have been considered to schedule the works. Then, we take stock of the advance state about undertaken works.

\section{Introduction}

En 1998, le Conseil Général des Pyrénées-Atlantiques a décidé d'entreprendre le diagnostic des ouvrages portuaires départementaux. Les ports 
concernés sont, du nord au sud : Biarritz, Guéthary, Saint-Jean-de-Luz / Ciboure, Socoa et Hendaye. L'étude inclut les ouvrages intérieurs (quais, débarcadères, etc.) et les ouvrages de protection extérieurs (digues, jetées, épis, etc.). Elle est découpée en trois phases :

1. Diagnostic initial incluant les ouvrages (inspections visuelles, état des ouvrages, relevé des désordres, étude des documents en archive, etc.) et les sollicitations auxquelles ils sont soumis (houles, vents, courants etc.).

2. Définition des travaux de réhabilitation et confortement (méthodes de réparation, chiffrages en coûts d'ordre, délais)

3. Etablissement du programme pluriannuel des réparations, en concertation avec le Maître d'Ouvrage, et en tenant compte des urgences et des coûts.

C'est l'étude des conditions d'exposition des ouvrages à la houle et des critères d'établissement du programme de réparation qui sont développée ici.

\section{$\underline{\text { 2. La houle et les conditions d'exposition des ouvrages }}$}

Les caractéristiques géomorphologiques du Golfe de Gascogne engendrent, aux confins de la France et de l'Espagne, une concentration de l'énergie véhiculée par les états de mer formés sur l'Atlantique Nord. Il en résulte que le littoral du département des Pyrénées Atlantiques est exposé aux houles les plus sévères que connaissent les côtes de France métropolitaine.

L'établissement d'un programme de réparations d'ouvrages bâtis au cours des trois derniers siècles passe donc par la vérification et l'éventuelle requalification des houles de projet prises en compte lors de leur construction et des sollicitations qui en résultent. Compte tenu de la complexité des évolutions des états de mer et des focalisations locales de l'énergie, la détermination des houles dimensionnantes sur chacun des sites de l'étude a rendu nécessaire la mise en œuvre d'une méthodologie adaptée qui s'articule en trois phases :

- la détermination des houles au large dans le Golfe de Gascogne,

- l'étude des évolutions des états de mer jusqu'à la côte,

- l'étude locale du transfert des états de mer jusqu'aux ouvrages sur chaque site.

Les sollicitations subies par chaque ouvrage ont ensuite été évaluées avec des formulations empiriques adaptées (Sainflou, Ito, Minikin, Hudson ...) et analysées à la lumière de son état actuel. Les formules les plus pertinentes ont également été appliquées à l'envers pour évaluer les sollicitations et done les houles les plus sévères auxquelles chaque ouvrage est susceptible de résister.

Cette analyse a ensuite été rapprochée des données historiques (construction, réparation, modification) de l'ouvrage retrouvées dans les archives. Pour les ouvrages intérieurs des ports, les sollicitations ont été déterminées sur la base des phénomènes hydrodynamiques observés localement. 


\section{VII ${ }^{\text {èmes }}$ Journées Nationales Génie Civil - Génie Côtier, Anglet, France, 15-17 Mai 2002}

\subsection{Détermination des houles au large}

Les houles au large ont été déterminées à partir des données de la littérature. Pour chaque source d'information, une zone pertinente a été définie.

- L'Atlas de climatologie océanique satellitaire « CLIOSat» (Etude MétéoMer).

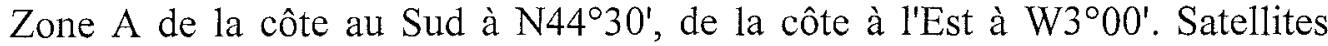
Geosat (11/1986 à 12/1989), Topex (10/1992 à 10/1998), ERS (04/1992 à 12/1998). Mesures altimétriques: $H_{\mathrm{s}}$ toutes incidences.

Zone $\mathrm{B}$ de la côte au Sud à $\mathrm{N}^{\circ} 6^{\circ} 30^{\prime}$ et de la côte à l'Est à $\mathrm{W} 7^{\circ} 30^{\prime}$. Satellites ERS1 et ERS2 (03/1992 à 12/1996). SAR en mode vague : $H_{\mathrm{s}}, T_{\mathrm{p}}$, incidence $\beta$.

- L'Atlas de climatologie océanique du BMT «Global Wave Statistics ». Zone $\mathrm{C}$ : Aire 17 de $N 43^{\circ} 00^{\prime}$ à $\mathrm{N} 50^{\circ} 00^{\prime}$ et de la côte à l'Est à W8 $00^{\circ}$ '. Observations de 1854 à 1984.

Ces données générales ont été complétées par des observations locales :

- Les éléments climatologiques concernant les côtes de la France : Observations de la station météorologique du Cap Ferret 1951 à 1980 (MétéoFrance),

- Le catalogue de fiches synthétiques de mesures de houle (Bouée de Bayonne du 19/07/89 au 31/12/92 et du 19/07/89 au 25/07/94) (STNMTE),

Les caractéristiques des houles ainsi obtenues pour les secteurs d'Ouest à Nord Nord-Ouest sont rassemblées dans le tableau 1. Pour le secteur Nord NordOuest à Nord, le fetch est limité par les côtes de Vendée et de Bretagne entre 300 et $500 \mathrm{~km}$. Les houles de projet ont alors été obtenues avec les formules de Bretschneider 1973 à partir des statistiques relatives au vent (tableau 1). Ces résultats ont été recoupés avec ceux retenus dans des études antérieures récentes.

\begin{tabular}{|c|c|c|c|c|c|c|c|c|}
\hline & \multicolumn{4}{|c|}{ Ouest à Nord Nord-Ouest } & \multicolumn{4}{c|}{ Nord Nord-Ouest à Nord } \\
\hline Période de retour & $H_{\mathrm{s}}(\mathrm{m})$ & $T_{\mathrm{s}}(\mathrm{s})$ & $H_{\max }(\mathrm{m})$ & $T_{\max }(\mathrm{s})$ & $H_{\mathrm{s}}(\mathrm{m})$ & $T_{\mathrm{s}}(\mathrm{s})$ & $H_{\max }(\mathrm{m})$ & $T_{\max }(\mathrm{s})$ \\
\hline Mensuelle & 3.6 & $9.0-14.0$ & 6.6 & $10.5-16.5$ & 2.4 & $7.3-11.3$ & 4.4 & $8.5-13.4$ \\
\hline Annuelle & 6.7 & $13.0-17.0$ & 12.0 & $16.5-19.5$ & 4.5 & $10.5-13.8$ & 8.1 & $13.4-15.8$ \\
\hline Décennale & 8.7 & $14.5-18.5$ & 14.9 & $18.0-21.5$ & 5.8 & $11.7-15.0$ & 9.9 & $14.6-17.4$ \\
\hline centennale & 10.3 & $16.0-20.0$ & 17.5 & $20.0-23.5$ & 6.9 & $13.0-16.2$ & 11.7 & $16.2-19.0$ \\
\hline
\end{tabular}

Tableau 1 : Houles de projet au large des Pyrénées Atlantiques.

\subsection{Transfert des états de mer dans le fond du Golfe de Gascogne}

A l'approche de la côte, la houle se déforme sous les effets de divers phénomènes (propagation, amortissement, shoaling, réfraction, diffraction, réflexion) qui se manifestent plus ou moins en fonction des caractéristiques géographiques de la zone considérée. La relation entre la hauteur de la houle au large et la hauteur de la houle sur un site donné, doit alors être déterminée au moyen d'un modèle d'approche prenant en compte l'évolution du train d'onde du large vers la côte ; les réflexions sur la côte étant d'un intérêt mineur.

Pour transférer les houles du large jusqu'à la côte, un modèle général, couvrant l'ensemble du littoral des Pyrénées Atlantiques, a été construit. Il couvre 
une zone de $50 \mathrm{~km}$ de côté allant de Cap Breton au Nord-Est jusqu'à San Sebastian au Sud-Ouest. Ce modèle, fondé sur la théorie des rayons en réfraction pure, prend en compte l'ensemble des phénomènes hormis la diffraction de la houle. Il permet d'obtenir les caractéristiques de la houle devant les sites de l'étude, en fonction du secteur d'incidence et de la période de retour. La figure 1 présente le modèle général pour une incidence d'Ouest et une période de 15 secondes.

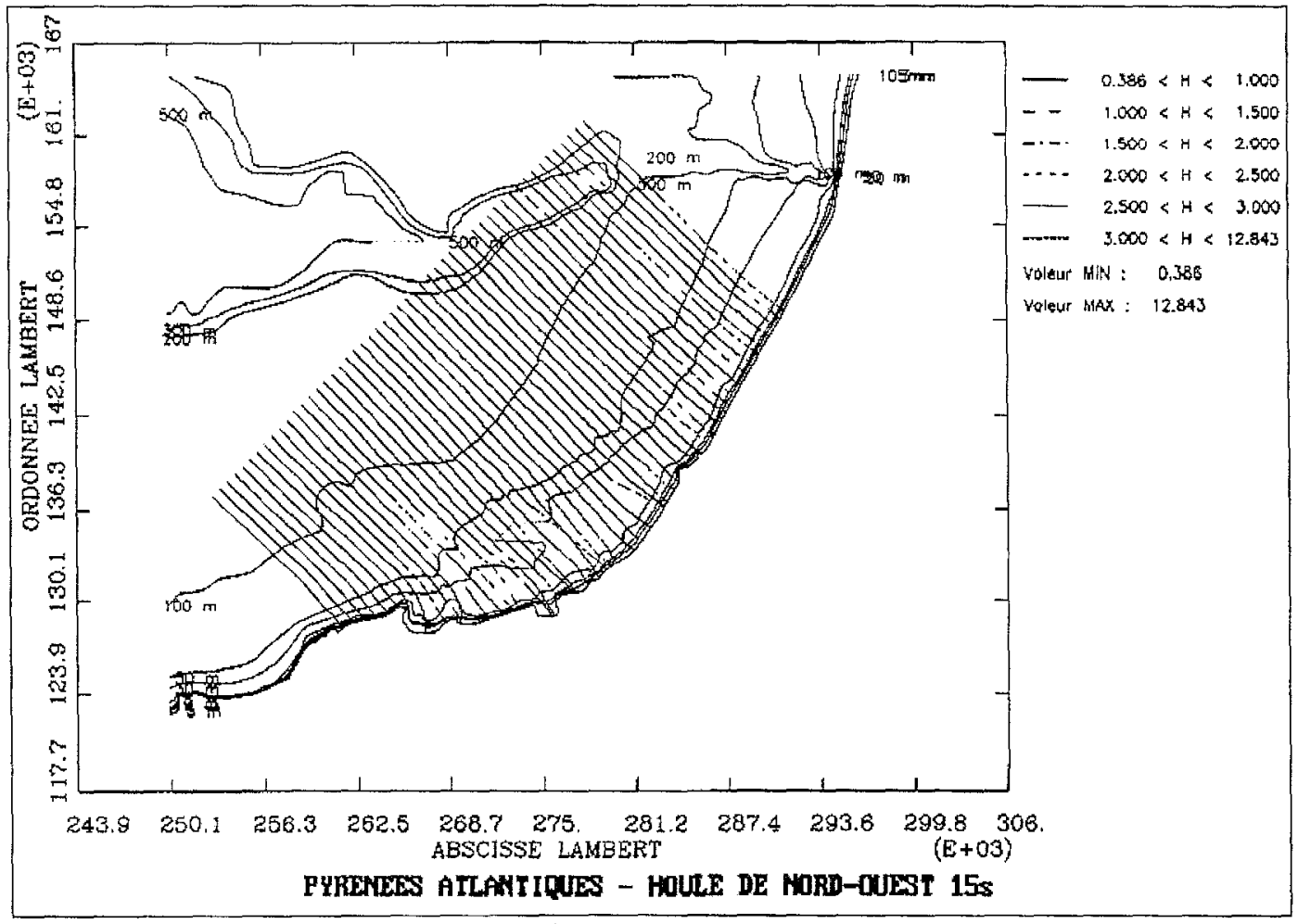

Figure 1 : Modèle général d'approche des Pyrénées Atlantiques

Figure 1: General wave model of Pyrénées Atlantiques

Le modèle d'approche ne prend en compte que l'amortissement de la houle par frottement sur le fond. La hauteur de la houle au large a donc été choisie pour correspondre aux plus fortes houles.

\begin{tabular}{|c|c|c|c|c|c|}
\hline Incidence & Bayonne & Biarritz & Guéthary & St Jean de Luz & Hendaye \\
\hline N & 0.83 & 0.86 & 0.55 & 0.93 & 1.03 \\
N NW & 0.89 & 0.96 & 0.63 & 0.98 & 1.00 \\
NW & 1.00 & 0.97 & 1.03 & 1.04 & 1.03 \\
W NW & 0.95 & 0.97 & 0.95 & 1.25 & 0.76 \\
W & 0.95 & 1.05 & 0.79 & 0.90 & 0.62 \\
\hline
\end{tabular}

Tableau 2 : Coefficients moyens de transmission sur les différents sites.

Les coefficients de transmission obtenus sur la côte, par une profondeur donnée caractéristique de chaque site, ont été moyennés sur une distance de l'ordre d'un kilomètre de longueur de littoral. La sensibilité du résultat à cette longueur a été analysée. Ils varient de 0.55 à 1.25 selon les sites et selon les incidences. 


\subsection{Détermination des houles à la côte sur chaque site}

Etant données les caractéristiques géomorphologiques du littoral, les résultats du modèle d'approche global s'appliquent directement au site de Guéthary, aux ouvrages à l'entrée de la baie de Saint-Jean-de-Luz (digues de Sainte-Barbe, de l'Artha et de Socoa) et au site d'Hendaye (Epi de Socoburu).

Ce n'est, par contre, pas le cas pour le site du Port des Pêcheurs de Biarritz protégé par une semis d'îlots ni pour les sites du port de Saint-Jean-de-Luz / Ciboure et de Socoa protégés à l'intérieur de la Baie de Saint-Jean-de-Luz. Pour chacun d'eux, un modèle d'approche local, de 3 à $5 \mathrm{~km}$ de côté, a été réalisé pour transférer les résultats du modèle général depuis la ligne bathymétrique des 20 mètres CM (Biarritz) ou 30 mètres CM (Saint-Jean-de-Luz) jusqu'aux ouvrages.

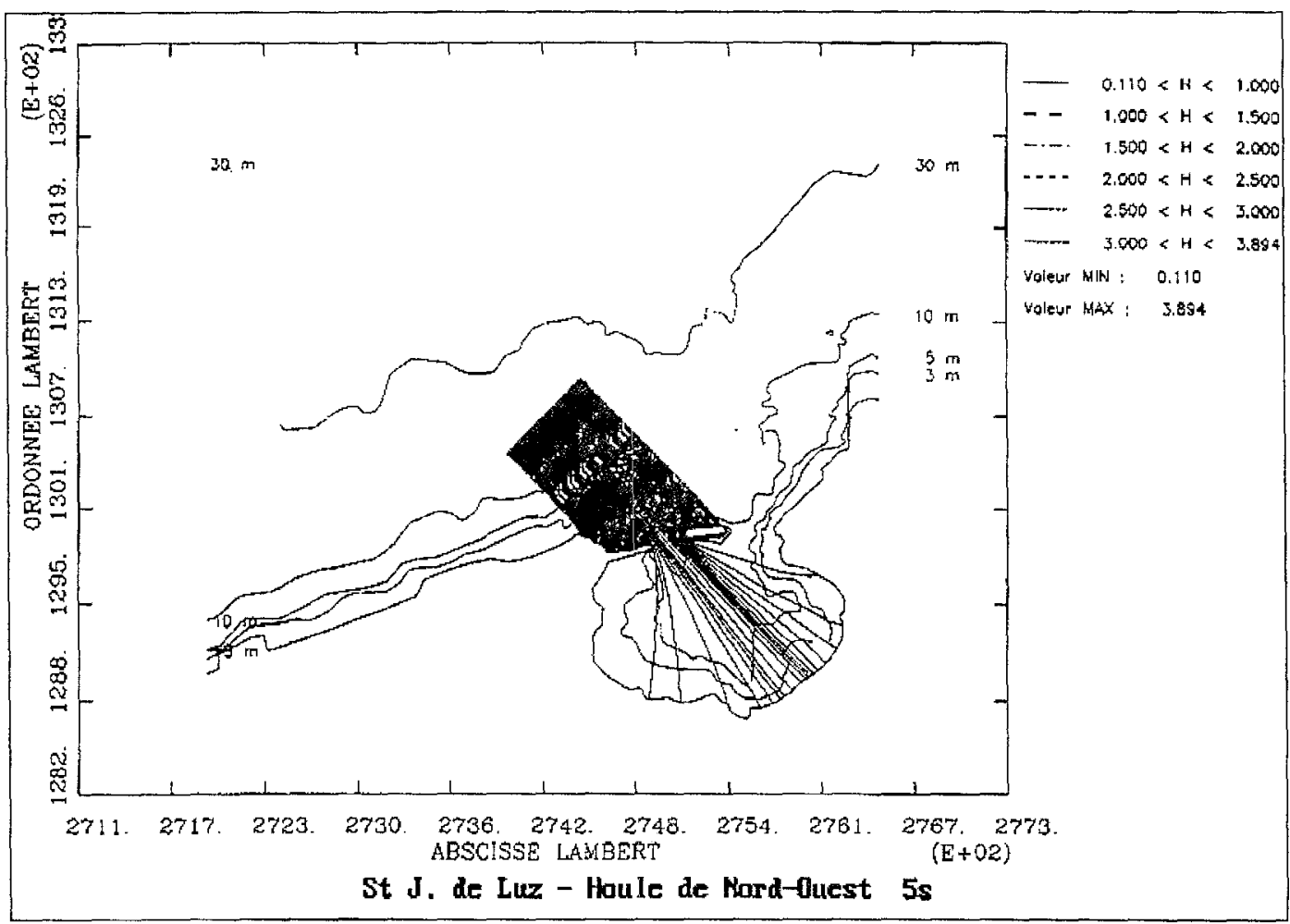

Figure 2 : Modèle local d'approche de Saint-Jean-de-Luz

Figure 2: Local wave model of Saint-Jean-de-Luz

Pour des raisons de clarté, à Saint-Jean-de-Luz, les plans de houle ont été réalisés séparément pour chacune des deux passes de part et d'autre de l'Artha. La figure 2 présente le modèle local de Saint-Jean-de-Luz pour une incidence d'Ouest et une période de 5 secondes à l'entrée de la passe de Socoa.

Les modèles d'approche locaux, combinés au modele d'approche général ont permis de définir les houles à prendre en compte devant les ouvrages des sites à géographie complexes. A noter que les résultats obtenus à Saint-Jean-de-Luz et Biarritz ont ultérieurement servi d'entrée à des modèles d'agitation. 


\section{Diagnostic et travaux de confortement}

L'inspection de l'ensemble des ouvrages a mis en évidence de nombreux problèmes à traiter :

- Confortement de glissements de terrains, traitements d'affouillements et injections de cavités plus ou moins importantes dans les fondations,

- Rempiétement de quais et jetées, confortement de rideaux de palplanches,

- Cavités et fissures dans les ouvrages,

- Renforcement de parements et de couronnements, pose de clameaux, rejointoiements de maçonneries,

- Confortement de Musoirs,

- Recharge et réarrangement d'enrochements en partie courante et en musoirs,

- Equipements de quai à rénover (échelles, caniveau technique, etc.),

- Amélioration de protection cathodique.

\section{Proposition des priorités d'intervention}

Trois critères ont été pris en compte pour définir des priorités d'intervention : le type d'ouvrage, le type de dégradation ou de rupture et les enjeux (tableau 3). Une valeur de 1 à 3 est affectée à la priorité de chaque critère (PCO, PCR et PCE). Une note de synthèse permet d'établir une priorité globale $\mathrm{PG}$ et de recommander la période d'intervention optimale au cours de la décennie à venir (tableau 4). Dans des cas de désordres multiples, la priorité globale peut prendre deux valeurs en considérant les urgences respectives de ces désordres ( $P G=1$ sur une partie de l'ouvrage et $P G=2$ ou 3 sur d'autres parties de l'ouvrage où les travaux sont moins urgents.

\begin{tabular}{|c|c|c|c|c|c|c|c|c|}
\hline & \multicolumn{2}{|c|}{ Type d'ouvrage } & \multicolumn{3}{|c|}{ Type de dégradation ou rupture } & \multicolumn{3}{|c|}{ Enjeux } \\
\hline Priorité 1 & $\begin{array}{l}\text { Ouvrage de } \\
\text { protection } \\
\text { Extérieure }\end{array}$ & & $\begin{array}{l}\text { Menace de } \\
\text { ruine } \\
\text { immédiate }\end{array}$ & & & Graves & & \\
\hline Priorité 2 & $\begin{array}{l}\text { Ouvrage de } \\
\text { protection } \\
\text { extérieure }\end{array}$ & $\begin{array}{l}\text { Ouvrage } \\
\text { portuaire } \\
\text { intérieur }\end{array}$ & & $\begin{array}{c}\text { Risque de } \\
\text { ruine ou dégra- } \\
\text { dation rapide }\end{array}$ & & & Modérés & \\
\hline Priorité 3 & & $\begin{array}{l}\text { Ouvrage } \\
\text { portuaire } \\
\text { Intérieur }\end{array}$ & & & $\begin{array}{l}\text { Dégradation } \\
\text { progressive } \\
\text { assez lente }\end{array}$ & & & Faibles \\
\hline & \multicolumn{2}{|c|}{$\mathrm{PCO}$} & \multicolumn{3}{|c|}{ PCR } & \multicolumn{3}{|c|}{ PCE } \\
\hline
\end{tabular}

Tableau 3 : Définition des priorités en fonction des différents critères

La priorité selon le type d'ouvrage est désignée par PCO. Sa valeur a été attribuée en distinguant deux grands types d'ouvrages:

- Les ouvrages de protection extérieurs, classés, selon leur degré d'exposition à la houle, soit en priorité 1 (digue de l'Artha qui protège la baie de St-Jean-deLuz, digue d'enclôture du port des pêcheurs de Biarritz), soit en priorité 2 (Jetée du quai de l'Infante du port de St-Jean-de-Luz).

- Les ouvrages portuaires intérieurs, en principe bien abrités, sont classés en priorité 3 (quais du port de St-Jean-de-Luz, quai Pêche du port d'Hendaye). 
La priorité selon le type de dégradation ou rupture est désignée par PCR Sa valeur a été attribuée en distinguant trois états :

- Lorsque la ruine est constatée au moment du diagnostic (perré sous le quai Pascal Elissalt à St-Jean-de-Luz, mur de Trinka à Socoa), la priorité 1 s'impose. Elle a également été retenue lorsque le risque de ruine se révèle imminent (digue de Gamaritz) ou peut survenir brutalement sans prévenir (rideau de palplanches du quai de Larraldénia à Ciboure).

- Lorsque la ruine ou la progression des dégradations peuvent survenir rapidement, sans être pour autant brutale, laissant ainsi le temps de mettre l'ouvrage en sécurité, la priorité 2 a été adoptée (quai Maurice Ravel, ouvrages de défense de la baie de St-Jean-de-Luz usés et érodés par l'agression répétée de la houle).

- Lorsque la dégradation est lente et peut faire l'objet d'un suivi dans le temps, la priorité a été fixée à 3 (quais sur pieux de St-Jean-de-Luz qui subissent la pénétration, l'attaque et la progression irréversible, mais lente, des chlorures).

\begin{tabular}{|c|c|c|c|c|c|c|c|}
\hline \multirow{3}{*}{ PORTS ET OUVRAGES } & \multicolumn{4}{|c|}{ Priorités } & \multicolumn{3}{|c|}{ Programmation annuelle proposée } \\
\hline & & & & & \multirow{2}{*}{$\begin{array}{|rr|}2000 & 2001 \\
\text { Priorité } 1\end{array}$} & \multirow{2}{*}{\begin{tabular}{|c|}
2002200320042005 \\
Priorité 2
\end{tabular}} & \multirow{2}{*}{\begin{tabular}{|c|}
2006200720082009 \\
Priorité 3
\end{tabular}} \\
\hline & PCO & PCE & PCR & $P G$ & & & \\
\hline \multicolumn{8}{|l|}{ BIARRITZ } \\
\hline Digue de Gamaritz & 1 & 1 & 1 & 1 & & & \\
\hline Digue d'enclôture & 1 & 1 & 2 & 1 & & & \\
\hline GUETHARY & & & & & & & \\
\hline Digue d'enclôture & 1 & 2 & 2 & 2 & & & \\
\hline \multicolumn{8}{|l|}{ St-JEAN-de-LUZ/CIBOURE } \\
\hline Quai Maurice Ravel & 2 & 1 & 2 & 2 & & & \\
\hline Jetée du quai de I'Infante & 2 & 1 & 1 & 1 & & & \\
\hline Bassin Larraldénia (SNCF) & 3 & 1 & 1 & 1,2 & & & \\
\hline Bassin Larraldénia (Ciboure) & 3 & 2 & 3 & 3 & & & \\
\hline Quai François Turnaco & 3 & 2 & 3 & 3 & & & 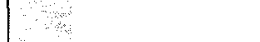 \\
\hline Quai Pascal Elissalt (perré) & 3 & 1 & 1 & 1 & & & \\
\hline Quai Pascal Elissalt & 3 & 1 & 3 & 1,2 & & & \\
\hline Quai de Verdun & 3 & 2 & 3 & 3 & & & \\
\hline Quai Général Leclerc & 3 & 2 & 3 & 1,3 & 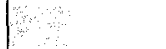 & & \\
\hline \multicolumn{8}{|l|}{ SOCOA } \\
\hline Digue de Trinka & 1 & 1 & 1 & 1 & & & \\
\hline Quai Sud & 2 & 1 & 3 & 2 & & 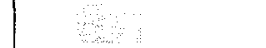 & \\
\hline Digue de la route maritime & 3 & 2 & 3 & 3 & & & \\
\hline Digue des douaniers & 3 & 3 & 3 & 3 & & & ... \\
\hline Digue du marégraphe & 2 & 2 & 3 & 2 & & a & \\
\hline \multicolumn{8}{|l|}{ BAIE DE St-JEAN-DE-LUZ } \\
\hline Digue de l'Artha & 1 & 1 & 2 & 1 & & & \\
\hline Digue de Socoa & 1 & 1 & 2 & 1 & & & \\
\hline Digue de Sainte-Barbe & 1 & 1 & 2 & 1 & & & \\
\hline \multicolumn{8}{|l|}{ HENDAYE } \\
\hline Epi de Socoburu & 1 & 2 & 3 & 2 & & & \\
\hline Quai Pêche & 3 & 2 & 3 & 2,3 & & & \\
\hline
\end{tabular}

PCO : Priorité selon le type d'ouvrage, PCE : Priorité selon les enjeux, PCR : Priorité selon le type de dégradation ou de rupture, $\mathrm{PG}$ : Priorité globale

Tableau 4 : Priorité globale en fonction des différents critères

La priorité selon les enjeux est désignée par PCE. L'attribution de sa valeur nécessite l'analyse des conséquences qu'entraînerait la ruine de chaque 
ouvrage. Elle est complexe et fait intervenir des notions de risques de natures très différentes.

- Les enjeux sont très élevés (priorité 1) pour des ouvrages de protection, comme dans la baie de St-Jean-de-Luz où leur ruine pourrait engendrer une submersion partielle de la ville.

- Les enjeux sont également importants (priorité 1) pour des ouvrages indispensables au maintien de l'activité économique comme le quai Pascal Elissalt qui supporte la criée du port de St-Jean-de-Luz.

- Les enjeux peuvent être modérés, voire même faibles, (priorité 2) pour d'autres ouvrages comme les quais du Maréchal Leclerc et de Verdun de StJean-de-Luz, les ouvrages du port de Guéthary ou ceux du port d'Hendaye.

- La digue des douaniers du port de Socoa, dont l'intérêt est essentiellement celui du patrimoine historique, peut être classée en priorité 3.

\section{$\underline{\text { 5. Mise en cuvre du programme de confortement }}$}

Depuis cette étude, les travaux entrepris sur les ports départementaux par le Conseil Général des Pyrénées Atlantiques ont suivi les prescriptions chronologiques établies par BRL Ingénierie :

- 2000 : rempiètement de la jetée du port de Saint Jean de Luz : vibrofonçage de palplanches, bétonnage. Des travaux de dragage, balisage et éclairage sont en cours pour améliorer la navigabilité dans le chenal, très exposé à la houle.

- Fin 2000 - Début 2001 : Injections de cavité et rejointoiement de la digue du Marégraphe à Socoa. L'attention particulière portée à la couleur des joints a permis une parfaite intégration de la digue rénovée dans le site.

- Septembre - Octobre 2001 : Confortement de la digue de Sainte Barbe. La digue ayant beaucoup souffert lors de dernières tempêtes, l'intervention devenait très urgente. L'inaccessibilité à la digue (chemin des falaises effondré) rendant le chantier très difficile et dangereux, le bétonnage a été réalisé en l'hélicoptère, comme pour un chantier en montagne.

Les efforts du Conseil Général des Pyrénées Atlantiques en matière d'entretien et d'investissement se poursuivront les prochaines années avec, par exemple, comme travaux projetés en 2002 et 2003 :

- Réfection complète du quai Pascal Elissalt (criée de St Jean de Luz / Ciboure),

- Dragage d'entretien du port,

- Réfection de la digue de Socoa.

\section{Conclusion}

Ces programmes pluriannuels reposent bien évidemment sur les conclusions et priorités établies par le diagnostic de 2000. Toutefois, l'évolution rapide des dégradations de certains ouvrages (quai Elissalt), ou les dégradations causées par les intempéries (Digue de Ste Barbe), obligent le Conseil Général à 
actualiser ses prévisions de réparations. La conduite de ce type d'étude montre la ferme volonté du Conseil général des Pyrénées Atlantiques de mettre en valeur le patrimoine portuaire départemental, et de l'entretenir et d'y investir.

\section{Références}

[1] Daguenet M. (1880 révisé en 1886) - Port de Biarritz - Rapport des Ponts et Chaussées

[2] Delure G. (1906) - Tournée d'inspection générale - Ports maritimes - Rapport des Ponts et Chaussées

[3] SOGREAH (1971) - Aménagement de la baie de Saint Jean de Luz, Etablissement d'esquisses de plages, rapport 10905

[4] Météorologie Nationale (1987) - Eléments climatologiques concernant les côtes de la France métropolitaine Fascicule V : de Chassiron à Cap Ferret Période 1951-1980

[5] STNMTE (1993) - Catalogue de fiches synthétiques de mesures de houle 